VOL. 24 (1981), 13-26.

\title{
ISOMETRIES OF MEASURABLE FUNCTIONS
}

\author{
Michael CAMBern
}

\begin{abstract}
Let $(X, \Sigma, \mu)$ be a $\sigma$-finite measure space and denote by $L^{\infty}(X, K)$ the Banach space of essentially bounded, measurable functions. $F$ defined on $X$ and taking values in a separable Hilbert space $K$. In this article a characterization is given of the linear isometries of $L^{\infty}(X, K)$ onto itself. It is shown that if $T$ is such an isometry then $T$ is of the form $(T(F))(x)=U(x)(\Phi(F))(x)$, where $\Phi$ is a set isomorphism of $\Sigma$ onto itself, and $U$ is a measurable operator-valued function such that $U(x)$ is almost everywhere an isometry of $K$ onto itself. It is a consequence of the proof given here that every isometry of $L^{\infty}(X, K)$ is the adjoint of an isometry of $L^{1}(X, K)$.
\end{abstract}

\section{Introduction}

Throughout this article $(X, \Sigma, \mu)$ will denote a $\sigma$-finite measure space, and the letter $K$ will represent a separable Hilbert space which may be either real or complex. We denote by $\langle\cdot, \cdot\rangle$ the inner product in $K$, and by $S$ the one-dimensional Hilbert space which is the scalar field associated with $K$.

A function $E$ from $X$ to $K$ will be called measurable if the scalar function $\langle E, e\rangle$ is measurable for each $e \in K$. Then for $1 \leq p \leq \infty$, we denote by $L^{p}(X, K)$ the Banach space of (equivalence classes of) measurable functions $F$ from $X$ to $K$ for which the norm

Received 14 January 1981. 


$$
\begin{aligned}
& \|F\|_{p}=\left\{\int\|F(x)\|^{p} d \mu\right\}^{1 / p}, p<\infty, \\
& \|F\|_{\infty}=\operatorname{ess} \sup \|F(x)\|
\end{aligned}
$$

is finite. $\left(\|\cdot\|_{p}\right.$ will denote the norm in $L^{p}(X, K)$ and $\|\cdot\|$ that in $K$.$) If F \in L^{P}(X, K)$, we define the support of $E$, which will be denoted by $\operatorname{supp}(F)$, to be the set $\{x \in X: F(x) \neq 0\}$.

Let $\left\{e_{1}, e_{2}, \ldots\right\}$ be some orthonormal basis for $K$. For $F \in L^{P}(X, K)$, we define the measurable coordinate functions $f_{n}$ by $f_{n}(x)=\left\langle E(x), e_{n}\right\rangle$. Then almost everywhere we have $\sum_{n}\left|f_{n}(x)\right|^{2} \ll \infty$, and $F(x)=\sum_{n} f_{n}(x) e_{n}$. Moreover, it is clear that each $f_{n}$ belongs to $L^{p}(X, S)$

In $\left[1\right.$, p. 178], Banach determined the isometries of ${ }_{L}^{P}(X, S)$, $1 \leq p<\infty, p \neq 2$, for the case in which $X$ is the unit interval and $\mu$ Lebesgue measure. Lamperti later obtained a complete description of the isometries of $L^{p}(X, S)$ for an arbitrary $\sigma$-finite measure space $(X, \Sigma, \mu)$, and the same values of $p,[8]$. The first such result for vector-valued functions was obtained in [2], where the surjective isometries of $L^{p}(X, K)$, for $1 \leq p<\infty, p \neq 2$, were characterized. This result was also established, via quite different methods, in [6] by Fleming and Jamison, and strengthened in [9] by Sourour, who replaced the Hilbert space $K$ by a separable Banach space $E$ having only trivial ${ }_{L}^{P}$-sumnands. In this article we investigate the surjective isometries of $L^{\infty}(X, K)$.

If $K$ is the one-dimensional Hilbert space $S$, then since $L^{\infty}(X, S)$ is isometrically isomorphic to $C(Y)$, where $y$ is the maximal ideal space of $L^{\infty}(X, S)$, a description of the isometries can be obtained through an application of the Banach-Stone theorem. And if $K$ is finite dimensional, it can be shown that $L^{\infty}(X, K)$ is isometrically isomorphic to $C(Y, K)$, the space of continuous functions on $Y$ to $K$, under the map 
$\sum_{n=1}^{N} f_{n} e_{n}+\sum_{n=1}^{N} \hat{f}_{n} e_{n}$, where $f \rightarrow \hat{f}$ is the Gelfand representation of $L^{\infty}(X, S)$. In this case the description we give in this article can also be obtained from what is known about isometries of spaces of continuous vector-valued functions [3]. However when $K$ is infinite dimensional, the continuity on $Y$ of the coordinate functions $\hat{f}_{n}$ no longer implies continuity for $\sum_{n} \hat{f}_{n} e_{n}$, and thus the problem requires different methods of approach.

A mapping $\Phi$ of $\Sigma$ onto itself, defined modulo null sets, is called a regular set isomorphism if it satisfies the properties

$$
\begin{aligned}
\Phi\left(A^{\prime}\right) & =[\Phi(A)]^{\prime}, \\
\Phi\left(\bigcup_{n=1}^{\infty} A_{n}\right) & =\bigcup_{n=1}^{\infty} \Phi\left(A_{n}\right),
\end{aligned}
$$

and

$$
\mu[\Phi(A)]=0 \text { if, and only if, } \mu(A)=0 \text { for all sets } A, A_{n} \text { in } \Sigma \text {. }
$$

(Throughout, $A^{\prime}$ will denote the complement of $A$. ) A regular set isomorphism induces a linear transformation, also denoted by $\Phi$, on the space of measurable scalar functions defined on $X$, which is characterized by $\Phi\left(\mathrm{X}_{A}\right)=\mathrm{X}_{\Phi(A)}$, where $\mathrm{X}_{A}$ is the characteristic function of the measurable set $A$. This process is described by Doob in [5, pp. 453-454].

Given a regular set isomorphism $\Phi$ of $\Sigma$ onto itself, and $F=\sum_{n} f_{n} e_{n}$ in $L^{p}(X, K)$, we define $\Phi(E)$ by the equation

$$
(\Phi(F))(x)=\sum_{n}\left(\Phi\left(f_{n}\right)\right)(x) e_{n}
$$

The fact that, in the case in which $K$ is infinite dimensional, the series on the right is indeed convergent in $K$ for almost all $x$ was established in $[2, p .10]$. One readily verifies that the definition of $\Phi(F)$ is independent of the choice of orthonormal basis for $K$.

We will use the fact that the set of extreme points of the unit ball in $L^{\infty}(X, K)$ consists of those elements $F$ such that $\|F(x)\|=1$ almost 
everywhere on $X$. Throughout the article, given $e \in K$, we denote by $\mathbf{e}$ that element of $L^{\infty}(X, K)$ which is constantly equal to $e$.

\section{Isometries}

Throughout, $T$ will denote a fixed isometry of $L^{\infty}(X, K)$ onto itself.

LEMMA 1. Let $E$ be an element of $L^{\infty}(X, K)$ with $\|E(x)\|=1$ almost everywhere. If $A \in \Sigma$ then $\operatorname{supp}\left(T\left(\mathrm{X}_{A} \cdot E\right)\right)$ and $\operatorname{supp}\left(T\left(\mathrm{X}_{A}, \cdot E\right)\right)$ are disjoint measurable sets whose union is almost everywhere equal to $X$. Moreover, $\left\|\left(T\left(\mathrm{X}_{A} \cdot E\right)\right)(x)\right\|$ is equal to one almost everywhere on $\operatorname{supp}\left(T\left(\chi_{A} \cdot E\right)\right)$

Proof. Note that since $E$ is an extreme point of the unit ball in $L^{\infty}(X, K)$, so is $T(E)$, and thus we have, almost everywhere,

$$
I=\|(T(E))(x)\|=\left\|\left(T\left(\chi_{A} \cdot E\right)\right)(x)+\left(T\left(\chi_{A}, \cdot E\right)\right)(x)\right\| .
$$

First suppose that $\left\|\left(T\left(X_{A} \cdot E\right)\right)(x)\right\| \neq I$ almost everywhere on $\operatorname{supp}\left(T\left(\mathrm{X}_{A} \cdot E\right)\right)$; that is, there is a measurable subset $B \subseteq \operatorname{supp}\left(T\left(\mathrm{X}_{A} \cdot E\right)\right)$ with $\mu(B)>0$ and $\left\|\left(T\left(\chi_{A} \cdot E\right)\right)(x)\right\|<1-\varepsilon_{1}$ for some $\varepsilon_{1}>0$ on $B$.

The set $\left\{x:\left\|\left(T\left(X_{A}, \cdot E\right)\right)(x)\right\|=I\right\}$ cannot intersect $\operatorname{supp}\left(T\left(X_{A} \cdot E\right)\right)$ in a set of positive measure. For $\left\|\mathrm{X}_{A} \cdot E \pm \mathrm{X}_{A}, \cdot E\right\|_{\infty}=1$ gives $\left\|\left(T\left(\mathrm{X}_{A} \cdot E\right)\right)(x) \pm\left(T\left(\mathrm{X}_{A}, \cdot E\right)\right)(x)\right\| \leq 1$ almost everywhere, and $\left(T\left(\mathrm{X}_{A}, \cdot E\right)\right)(x)$ is an extreme point of the unit ball of $K$ for all $x \in\left\{x:\left\|\left(T\left(X_{A}, \cdot E\right)\right)(x)\right\|=1\right\}$. Thus $\left\|\left(T\left(X_{A}, \cdot E\right)\right)(x)\right\|<1$ almost everywhere on $\operatorname{supp}\left(T\left(\mathrm{X}_{A} \cdot E\right)\right)$, so that we can find a subset $C$ of $B$ with $\mu(C)>0$ and $\left\|\left(T\left(x_{A}, \cdot E\right)\right)(x)\right\|<1-\varepsilon_{2}$ on $C$, for some $\varepsilon_{2}>0$.

Let $\varepsilon=\min \left\{\varepsilon_{1}, \varepsilon_{2}\right\}$. Then we certainly have $\left\|T\left(\chi_{A} \cdot E\right) \pm \varepsilon \cdot \chi_{C} \cdot E\right\|_{\infty} \leq 1$ and $\left\|T\left(\chi_{A}, \cdot E\right) \pm \varepsilon \cdot X_{C} \cdot E\right\|_{\infty} \leq 1$, so that $\left\|X_{A}(x) \cdot E(x) \pm\left(T^{-1}\left(\varepsilon \cdot \chi_{C} \cdot E\right)\right)(x)\right\| \leq 1$ and $\left\|\mathrm{X}_{A},(x) \cdot E(x) \pm\left(T^{-1}\left(\varepsilon \cdot \mathrm{X}_{C} \cdot E\right)\right)(x)\right\| \leq 1$ almost everywhere. But since $T^{-1}\left(\varepsilon \cdot X_{C} \cdot E\right)$ is not the zero element of $L^{\infty}(X, K)$, its support must meet 
either $A$ or $A^{\prime}$ in a set of positive measure, contradicting the fact that $x_{A}(x) \cdot E(x)$ is an extreme point of the unit ball of $K$ almost everywhere on $A$, and $\chi_{A},(x) \cdot E(x)$ is an extreme point of the unit ball of $K$ almost everywhere on $A^{\prime}$.

This contradiction shows that $\left\|\left(T\left(X_{A} \cdot E\right)\right)(x)\right\|=1$ almost everywhere on $\operatorname{supp}\left(T\left(\mathrm{X}_{A} \cdot E\right)\right)$. Similarly, $\left\|\left(T\left(\mathrm{X}_{A}, \cdot E\right)\right)(x)\right\|=1$ on $\operatorname{supp}\left(T\left(\mathrm{X}_{A}, \cdot E\right)\right)$. It is clear that $\operatorname{supp}\left(T\left(\mathrm{X}_{A} \cdot E\right)\right)$ and $\operatorname{supp}\left(T\left(\mathrm{X}_{A}, \cdot E\right)\right)$ are measurable. The fact that these sets are disjoint again follows since

$$
\left\|\left(T\left(X_{A} \cdot E\right)\right)(x) \pm\left(T\left(X_{A}, \cdot E\right)\right)(x)\right\| \leq 1
$$

almost everywhere, and $\left(T\left(X_{A} \cdot E\right)\right)(x)$ is an extreme point of the unit ball in $K$ for all $x \in \operatorname{supp}\left(T\left(\mathrm{X}_{A} \cdot E\right)\right)$. Finally, the union of $\operatorname{supp}\left(T\left(\mathrm{X}_{A} \cdot E\right)\right)$ and $\operatorname{supp}\left(T\left(\mathrm{X}_{A}, \cdot E\right)\right)$ is, by $(1)$, equal to $X$.

LEMMA 2. With $E$ as in Lemma 1 define, for $A \in \Sigma$, $\Phi(A)=\operatorname{supp}\left(T\left(\mathrm{X}_{A} \cdot E\right)\right)$. Then $\Phi$ is a regular set isomorphism of $\Sigma$ onto itself.

Proof. It follows immediately from Lemma 1 that for $A \in \Sigma$,

$$
\Phi\left(A^{\prime}\right)=[\Phi(A)]^{\prime} \text {. }
$$

Note that $\mu(A) \neq 0$ if and only if $\chi_{A} \cdot E \neq 0$ in $L^{\infty}(X, K)$ which is true if and only if $T\left(X_{A} \cdot E\right) \neq 0$ in $L^{\infty}(X, K)$ and this holds if and only if $\mu[\Phi(A)]>0$. Thus trivially we have

$$
\mu[\Phi(A)]=0 \text { if, and only if, } \mu(A)=0 \text {. }
$$

Now suppose that $A$ and $B$ are disjoint measurable sets. Since $\left\|\left(T\left(\mathrm{X}_{A} \cdot E\right)\right)(x) \pm\left(T\left(\mathrm{X}_{B} \cdot E\right)\right)(x)\right\| \leq 1$ almost everywhere and $\left(T\left(\mathrm{X}_{A} \cdot E\right)\right)(x)$ is an extreme point of the unit ball in $K$ for all $x \in \operatorname{supp}\left(T\left(\mathrm{X}_{A} \cdot E\right)\right), \Phi(A)$ and $\Phi(B)$ must be almost everywhere disjoint. Thus if $B$ is a measurable subset of the measurable set $A$, then $B$ and $A^{\prime}$ are disjoint so that $\Phi(B)$ and $\Phi\left(A^{\prime}\right)$ are disjoint. Hence $B \subseteq A$ implies that $\Phi(B) \subseteq \Phi(A)$. It is easily seen that the reverse implication is also true: $\Phi(B) \subseteq \Phi(A)$ implies that $B \subseteq A$. The sentence before last also implies that $A$ and 
$B$ are disjoint if and only if $\Phi(A)$ and $\bar{\varphi}(B)$ are disjoint.

Next assume that $\left\{A_{1}, A_{2}, \ldots\right\}$ is a pairwise disjoint sequence of measurable sets and let $A=\bigcup_{n=1}^{\infty} A_{n}$. Then since $A_{n} \subseteq A$ for all $n$, we have $\Phi\left(A_{n}\right) \subseteq \Phi(A)$ for all $n$, so that $\bigcup_{n=1}^{\infty} \Phi\left(A_{n}\right) \subseteq \Phi(A)$. Set $B=\Phi(A)-\bigcup_{n=1}^{\infty} \Phi\left(A_{n}\right)$. We would like to show that $\mu(B)=0$. To this end we first show that $\Phi$ maps $\Sigma$ onto itself.

Thus suppose that $B$ is any measurable set. We have

$$
T(E)=\chi_{B} \cdot T(E)+\chi_{B^{\prime}} \cdot T(E)
$$

so that

$$
E=T^{-1}\left[\chi_{B} \cdot T(E)\right]+T^{-1}\left[\chi_{B}, T(E)\right]
$$

By Lemma 1 (interchanging the roles of $T$ and $T^{-1}, A$ and $B, E$ and $T(E)$ ), the two elements on the right in the last equation have disjoint supports, and have norm equal to one almost everywhere on their respective supports. Thus $T^{-1}\left[X_{B} \cdot T(E)\right]$ is of the form $X_{C} \cdot E$ for some $C \in \Sigma$, and hence

$$
T\left(\chi_{C} \cdot E\right)=\chi_{B} \cdot T(E)
$$

which says that $\Phi(C)=B$. Thus $\Phi$ is onto.

$$
\text { Now with } B=\Phi(A)-\bigcup_{n=1}^{\infty} \Phi\left(A_{n}\right) \text {, take } C \in \Sigma \text { with } \Phi(C)=B \text {. By }
$$

what was established in the second paragraph of this proof, we must have $C \subseteq A$ in this instance. Thus if we suppose that $B$, hence $C$, has positive measure, then, for some $n, C$ meets $A_{n}$ in a set of positive measure. But $\Phi(C)$ and $\Phi\left(A_{n}\right)$ are disjoint, and this contradiction shows that we must have $\mu(B)=0$. Thus 


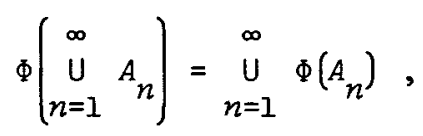

completing the proof of the lemma.

NOTE. Equation (2) tells us that for each $A \in \Sigma$,

$$
T\left(\chi_{A} \cdot E\right)=\chi_{\Phi(A)} \cdot T(E)
$$

From this it follows that for every $f \in L^{\infty}(X, S)$ we have

$$
T(f \cdot E)=\Phi(f) \cdot T(E) .
$$

LEMMA 3. If $E_{1}$ and $E_{2}$ are two pointwise orthogonal elements of $L^{\infty}(X, K)$ with $\left\|E_{j}(x)\right\|=1$ almost everywhere on $X, j=1,2$, then $T\left(E_{1}\right)$ and $T\left(E_{2}\right)$ are pointwise orthogonal for almost alz $x$.

Proof. Using the polarization identity [7, p. 274] (and assuming the space is complex), we have

$$
\begin{array}{r}
\left\langle\left(T\left(E_{1}\right)\right)(x), \quad\left(T\left(E_{2}\right)\right)(x)\right\rangle=\frac{1}{4} \sum_{n=1}^{4} i^{n}\left\|\left(T\left(E_{1}\right)\right)(x)+i^{n}\left(T\left(E_{2}\right)\right)(x)\right\|^{2} \\
=\frac{3}{4} \sum_{n=1}^{4} i^{n}\left\|\left(T\left(E_{1}+i^{n} E_{2}\right)\right)(x)\right\|^{2} .
\end{array}
$$

But the elements $E_{1}+i^{n} E_{2}, n=1,2,3,4$, are extreme points of the ball of radius $\sqrt{2}$ centered at the origin in $L^{\infty}(X, K)$. Hence $\left\|\left(T\left(E_{1}+i^{n_{2}}\right)\right)(x)\right\|=\sqrt{2}$ almost everywhere on $X$, so the inner product $\left(\left(T\left(E_{1}\right)\right)(x),\left(T\left(E_{2}\right)\right)(x)\right)$ is almost everywhere equal to zero.

If $K$ is a real Hilbert space, the corresponding polarization identity also gives the desired result.

LEMMA 4. Let $E_{1}$ and $E_{2}$ be as in Lemma 3 , and for $j=1,2$, let $\Phi_{j}$ be the regular set isomorphism of $\Sigma$ onto itself that is associated with $E_{j}$ as in Lemma 2. Then $\Phi_{1}=\Phi_{2}$.

Proof. Let $A \in \Sigma^{*}$ and consider 


$$
\begin{aligned}
\left(T\left(\mathrm{X}_{A} \cdot\left(E_{1}+E_{2}\right) / \sqrt{2}\right)\right)(x) & =(1 / \sqrt{2})\left(T\left(\mathrm{X}_{A} \cdot E_{1}\right)\right)(x)+(1 / \sqrt{2})\left(T\left(\mathrm{X}_{A} \cdot E_{2}\right)\right)(x) \\
& =(1 / \sqrt{2}) \mathrm{X}_{\Phi_{1}(A)}(x)\left(T\left(E_{1}\right)\right)(x)+(1 / \sqrt{2}) \mathrm{X}_{\Phi_{2}(A)}(x)\left(T\left(E_{2}\right)\right)(x)
\end{aligned}
$$

Now the left hand side of (3) is equal to

$$
\begin{aligned}
X_{\Phi_{1,2}}(A) & (x)\left(T\left(E_{1}+E_{2}\right) / \sqrt{2}\right)(x) \\
& =(1 / \sqrt{2}) X_{\Phi_{1,2}}(A)
\end{aligned}
$$

where $\Phi_{1,2}$ is the regular set isomorphism associated with $\left(E_{1}+E_{2}\right) / \sqrt{2}$ via Lemma 2. Thus, by the almost everywhere linear independence of $\left(T\left(E_{1}\right)\right)(x)$ and $\left(T\left(E_{2}\right)\right)(x)$ we have

$$
\chi_{\Phi_{1}(A)}(x)=\chi_{\Phi_{1,2}}(A)^{(x)=\chi_{\Phi_{2}}(A)}{ }^{(x)}
$$

almost everywhere, and hence $\Phi_{1}=\Phi_{2}$.

LEMMA 5. Suppose that $F_{1}, F_{2}$ are elements of $L^{\infty}(X, K)$ such that $F_{1}(x)$ and $F_{2}(x)$ are orthogonal in $K$ for almost all $x$. Then $\left.\left(T\left(F_{1}\right)\right)(x),\left(T\left(F_{2}\right)\right)(x)\right)=0$ almost everywhere.

Proof. First suppose that $F_{1}=g_{1} G_{1}$ and $F_{2}=g_{2} G_{2}$, where the $g_{j}$ are elements of $L^{\infty}(X, S)$ with $\operatorname{supp}\left(g_{j}\right)=\operatorname{supp}\left(F_{j}\right)$ and the $G_{j}$ are measurable vector functions such that $\left\|G_{j}(x)\right\|=1$ on $\operatorname{supp}\left(F_{j}\right)$, $j=1,2$. Let $A=\operatorname{supp}\left(E_{2}\right)-\operatorname{supp}\left(E_{1}\right)$. On $A$ we have $F_{2}(x)=\sum_{n} f_{n}(x) e_{n}$, the convergence being almost everywhere pointwise in $K$. Since $A=\underset{n}{\cup} A_{n}$, where $A_{n}=\left\{x \in A: f_{n}(x) \neq 0\right\}$ we can define a measurable vector function $H_{1}$ on $A$ by $H_{1}(x)=\overline{f_{2}(x)} e_{1}-\overline{f_{1}(x)} e_{2}$, for $x \in A_{1} \cup A_{2}$, and for $n>2, H_{1}(x)=\overline{f_{n}(x)} e_{1}-\overline{f_{1}(x)} e_{n}$ for $x \in A_{n}-\left(A_{1} \cup \ldots \cup A_{n-1}\right)$. Then $H_{1}(x)$ is almost everywhere orthogonal to $F_{2}(x)$ on $A$, and the vector function $J_{1}$ defined on $A$ by $J_{1}(x)=H_{1}(x) /\left\|H_{1}(x)\right\|, x \in A$, is such that $\left\|J_{1}(x)\right\|=1$ for $x \in A$ and 
$J_{1}(x)$ is pointwise orthogonal to $E_{2}(x)$ almost everywhere on $A$.

Similarly we can find a measurable vector function $J_{2}$ defined on $B=\operatorname{supp}\left(F_{1}\right)-\operatorname{supp}\left(F_{2}\right)$ such that $\left\|J_{2}(x)\right\|=1$ on $B$ and $\left\langle F_{1}(x), J_{2}(x)\right\rangle=0$ almost everywhere on $B$. We now define measurable vector functions $E_{1}$ and $E_{2}$ by

$$
\begin{aligned}
& E_{1}(x)= \begin{cases}G_{1}(x), & x \in \operatorname{supp}\left(F_{1}\right), \\
J_{1}(x), & x \in A, \\
e_{1}, & x \in X-\left(\operatorname{supp}\left(F_{1}\right) \cup \operatorname{supp}\left(F_{2}\right)\right),\end{cases} \\
& E_{2}(x)= \begin{cases}G_{2}(x), & x \in \operatorname{supp}\left(F_{2}\right), \\
J_{2}(x), & x \in B, \\
e_{2}, & x \in X-\left(\operatorname{supp}\left(F_{1}\right) \cup \operatorname{supp}\left(F_{2}\right)\right) .\end{cases}
\end{aligned}
$$

Then $\left\|E_{j}(x)\right\|=1$ and $\left\langle E_{1}(x), E_{2}(x)\right\rangle=0$ almost everywhere.

We can write $F_{j}=g_{j} E_{j}, j=1,2$, so that by Lemma 4 and the note following Lemma 2 , there exists a regular set isomorphism $\Phi$ of $\Sigma$ onto itself such that $T\left(E_{j}\right)=\Phi\left(g_{j}\right) T\left(E_{j}\right)$ for $j=1,2$. We thus have

$$
\begin{aligned}
\left\langle\left(T\left(E_{1}\right)\right)(x),\left(T\left(F_{2}\right)\right)(x)\right\rangle & =\left\langle\left(\Phi\left(g_{1}\right)\right)(x)\left(T\left(E_{1}\right)\right)(x),\left(\Phi\left(g_{2}\right)\right)(x)\left(T\left(E_{2}\right)\right)(x)\right\rangle \\
& =\left(\Phi\left(g_{1}\right)\right)(x)\left(\Phi\left(g_{2}\right)\right)(x)\left\langle\left(T\left(E_{1}\right)\right)(x),\left(T\left(E_{2}\right)\right)(x)\right\rangle=0
\end{aligned}
$$

almost everywhere by Lemma 3 .

Now since every $E \in L^{\infty}(X, K)$ can be written in the form $E=g G$, where $g \in L^{\infty}(X, S)$ with $\operatorname{supp}(g)=\operatorname{supp}(F)$ and $G$ is measurable with $\|G(x)\|=1$ almost everywhere on $\operatorname{supp}(F)$ (that is, let $g(x)=\|F(x)\|$ and $G(x)=E(x) /\|F(x)\|$ for $x \in \operatorname{supp}(F)$ ) the proof of the lemma is complete.

LEMMA 6. Let $\Phi$ be the regular set isomorphism of $\Sigma$ onto itself determined by $T\left(\chi_{A} \cdot e_{n}\right)=\chi_{\Phi(A)} T\left(\mathrm{e}_{n}\right)$, for $n=1,2, \ldots$ and $A \in \Sigma$, and denote also by $\Phi$ the corresponding linear transformation of measurable scalar functions. Then the map defined for $F \in L^{\infty}(X, K)$ by $F \rightarrow \Phi(F)$ is an isometry of $L^{\infty}(X, K)$ onto itself. 
Proof. It follows from Lemma 4, the separability of $K$, and the note following Lemma 2 that there exists a well defined regular set isomorphism $\Phi$ of $\Sigma$ onto itself such that for all $n=1,2, \ldots$ and all bounded measurable scalar functions $f, T\left(f \cdot e_{n}\right)=\Phi(f) \cdot T\left(\mathrm{e}_{n}\right)$. It is obvious that $\|\Phi(f)\|_{\infty}=\|f\|_{\infty}$ holds for all scalar simple functions $f$, and the fact that the same equality then holds for an arbitrary element $f$ of $L^{\infty}(X, S)$ follows easily from the fact that $\Phi$ preserves the almost everywhere convergence of sequences of measurable functions.

Now using [2, p. 10] and the fact that $\Phi$ preserves the set of positive elements of $L^{\infty}(X, S)$, it follows that for $F \in L^{\infty}(X, K)$, $\|(\Phi(F))(\cdot)\|=\Phi(\|F(\cdot)\|)$, where $\|F(\cdot)\|$ denotes the $L^{\infty}(X, S)$ element $g$ defined by $g(x)=\|F(x)\|$ for $x \in X$. Thus

$$
\|\Phi(F)\|_{\infty}=\text { ess } \sup \|(\Phi(F))(x)\|=\text { ess } \sup \Phi(\|F(x)\|) \text {. }
$$

But by the previous paragraph this last quantity is equal to ess $\sup \|F(x)\|=\|F\|_{\infty}$ so that the map is norm preserving, and it is clear that it is also surjective.

We now define the measurable operator function $U(x)$ by first defining $U(x)$ on the basis vectors $e_{n}$ of $K$ via the equation $U(x) e_{n}=\left(T\left(\mathrm{e}_{n}\right)\right)(x)$, and then extending $U(x)$ linearly to $K$. By Lemma 3, $\left\{\left(T\left(\mathrm{e}_{1}\right)\right)(x),\left(T\left(\mathrm{e}_{2}\right)\right)(x), \ldots\right\}$ is almost everywhere an orthonormal set in $K$, so that $U(x)$ is an isometry of $K$ into itself almost everywhere. It thus follows from Lemma 6 that for $F \in L^{\infty}(X, K)$, the map $E(\cdot) \rightarrow U(\cdot)(\Phi(F))(\cdot)$ is an isometry of $L^{\infty}(X, K)$ into itself.

In the following lemma we use the fact that, modulo the usual conjugate-linear identification of $K$ with its own dual space, $L^{\infty}(X, K)$ is the dual space of $L^{1}(X, K) \quad[4$, p. 282].

LEMMA 7. For $F=\sum_{n} f_{n} e_{n} \in L^{\infty}(X, K)$ define, for $N=1,2, \ldots$, $E_{N}=\sum_{n=1}^{N} f_{n} e_{n}$. Then $U(\cdot)\left(\Phi\left(F_{N}\right)\right)(\cdot)$ tends to $U(\cdot)(\Phi(F))(\cdot)$ in the weak * topology of $L^{\infty}(X, K)$.

Proof. The sequence $\left\{\Phi\left(F_{N}\right)\right\}$ converges almost everywhere to $\Phi(F)$ 
and is uniformly bounded in norm by $\|\Phi(F)\|_{\infty}=\|F\|_{\infty}$. Hence the sequence $\left\{U(\cdot)\left(\Phi\left(F_{N}\right)\right)(\cdot)\right\}$ converges almost everywhere to $U(\cdot)(\Phi(F))(\cdot)$, and is also uniformly bounded in norm by $\|F\|_{\infty}$. It follows that if $G \in L^{1}(X, K)$, then $G(\cdot) U(\cdot)\left(\Phi\left(F_{N}\right)\right)(\cdot)$ converges almost everywhere to $G(\cdot) U(\cdot)(\Phi(F))(\cdot)$, and is dominated by $\|F\|_{\infty}\|G(\cdot)\|$. Thus, by the dominated convergence theorem,

$$
\begin{aligned}
\left|\int G(x) U(x)\left(\Phi\left(F_{N}\right)\right)(x) d \mu-\int G(x) U(x)(\Phi(F))(x) d \mu\right| \\
\leq \\
\leq \int G(x) U(x)\left(\Phi\left(F_{N}\right)\right)(x)-G(x) U(x)(\Phi(F))(x) \| d \mu \\
\rightarrow 0 \text { as } N \rightarrow \infty .
\end{aligned}
$$

LEMMA 8. Let $v$ be the measure defined on $\Sigma$ by $\nu(A)=\mu\left[\Phi^{-1}(A)\right]$, for $A \quad \Sigma$, and let $h=\frac{d \nu}{d \mu}$. Define $R$ mapping $L^{1}(X, K)$ to itself by $(R(G))(x)=U(x) h(x)(\Phi(G))(x)$, for $G \in L^{1}(X, K)$. Then $R$ maps $L^{l}(X, K)$ isometrically onto itself and $T=R^{*^{-1}}$.

Proof. First note that, by the definition of a regular set isomorphism, $\nu$ is absolutely continuous with respect to $\mu$ and that, for $\dot{A} \in \Sigma$, we have

$$
\mu(A)=\nu[\Phi(A)]=\int_{\Phi(A)} d \nu=\int_{\Phi(A)} \frac{d \nu}{d \mu} d \mu=\int_{\Phi(A)} h d \mu
$$

Moreover, by [9, p. 283] the mapping $R$ defined above is an isometry of $L^{1}(X, K)$ into itself.

If $G=\sum_{n} g_{n} e_{n} \in L^{I}(X, K)$, then the sequence $\left\{G_{N}\right\}$, where for $N=1,2, \ldots$ and $x \in X, G_{N}(x)=\sum_{n=1}^{N} g_{n}(x) e_{n}$, converges almost everywhere to $G$ and is dominated by $\|G\|$. Thus, by the dominated convergence theorem, finite sums of the form $G_{N}=\sum_{n=1}^{N} g_{n} e_{n}$ are dense in $L^{l}(X, K)$, and it is clear that we still have a dense set if we restrict the coordinate functions $g_{n}$ to be scalar simple functions. Moreover, it 
is also clear that $L^{\infty}(X, K)$ elements of the form $H(x)=\sum_{n} s_{n}(x) e_{n}$, where the $s_{n}$ are scalar simple functions, are dense in $L^{\infty}(X, K)$.

Thus suppose we have two such elements $G_{N} \in L^{1}(X, K)$ and $H \in L^{\infty}(X, K)$. We may suppose that $G_{N}=\sum_{i=1}^{N} \sum_{j=1}^{n} c_{i j} x_{A_{j}} e_{i}$ and $H=H_{1}+H_{2}$, where $H_{1}=\sum_{i=1}^{N} \sum_{j=1}^{n} d_{i j} x_{A_{j}} e_{i}, H_{2}=\sum_{i=N+1}^{\infty} s_{i} e_{i}$, the $c_{i j}, d_{i j}$ are scalars, and the $A_{j}$ are pairwise disjoint measurable sets. We thus have

$$
\int\left\langle G_{N}, H\right\rangle d \mu=\int\left(\sum_{i=1}^{N} \sum_{j=1}^{n} c_{i j} \bar{d}_{i j} x_{A_{j}}\right) d \mu=\sum_{i=1}^{N} \sum_{j=1}^{n} c_{i j} \bar{d}_{i j} \mu\left(A_{j}\right),
$$

But by (4) this latter quantity is equal to

$$
\begin{aligned}
\sum_{i=1}^{N} \sum_{j=1}^{n} c_{i j} \bar{d}_{i j} \int_{\Phi\left(A_{j}\right)} & h d \mu \\
& =\int\left\langle\sum_{i=1}^{N} \sum_{j=1}^{n} h c_{i j} x_{\Phi}\left(A_{j}\right) T\left(\mathrm{e}_{i}\right), \sum_{i=1}^{N} \sum_{j=1}^{n} d_{i j} \chi_{\Phi}\left(A_{j}\right)^{T}\left(\mathrm{e}_{i}\right)\right\rangle d \mu \\
& =\int\left(U(x) h(x)\left(\Phi\left(G_{N}\right)\right)(x),\left(T\left(H_{1}\right)\right)(x)\right) d \mu
\end{aligned}
$$

and since by Lemma $5,\left(T\left(H_{2}\right)\right)(x)$ is almost everywhere orthogonal in $K$ to $T\left(\mathbf{e}_{i}\right), 1 \leq i \leq N$, this last integral equals

$$
\begin{aligned}
\int\left(U(x) h(x)\left(\Phi\left(G_{N}\right)\right)(x),\left(T\left(H_{1}\right)\right)(x)\right. & \left.+\left(T\left(H_{2}\right)\right)(x)\right) d \mu \\
= & \int\left(R\left(G_{N}\right), T(H)\right) d \mu=\int\left(G_{N}, R^{*} \circ T(H)\right) d \mu .
\end{aligned}
$$

Here $R^{*}$ is, again modulo the conjugate-linear identification of $K$ with its own dual, the Banach space adjoint of $R$ [2, p. 11].

Thus for each $H$ in $L^{\infty}(X, K)$ of the form considered, we have shown that the linear functionals on $L^{\mathcal{L}}(X, K)$ determined by $H$ and $\left(R^{*} \circ T\right)(H)$ agree when evaluated at all elements $G_{N}$ belonging to a dense 
subset of $L^{1}(X, K)$. Hence, for such $H$, we have $\left(R^{*} \circ T\right)(H)=H$; and since this equality holds for all $H$ belonging to a dense subset of $L^{\infty}(X, K)$, we have $R^{*}=T^{-1}$ and $R^{*^{-1}}=T$. Note that this implies that $R$ is actually an isometry of $L^{l}(X, K)$ onto itself $[10$, p. 226].

THEOREM. FOP $F \in L^{\infty}(X, K),(T(F))(x)=U(x)(\Phi(F))(x)$.

Proof. Let $F=\sum_{n} f_{n} e_{n}$ belong to $L^{\infty}(X, K)$, and for $N=1,2, \ldots$ set $F_{N}=\sum_{n=1}^{N} f_{n} e_{n}$. Then by an argument exactly analogous to that given in the proof of Lemma $7, F_{N}+F$ in the weak * topology of $L^{\infty}(X, K)$. Since adjoints of maps continuous with respect to the norm topology remain continuous with respect to the weak * topology, we have $T\left(F_{N}\right)=R^{*-1}\left(F_{N}\right) \rightarrow R^{*-1}(F)=T(F)$ weak *. But by Lemma 7 , $T\left(F_{N}\right)=U(\cdot)\left(\Phi\left(E_{N}\right)\right)(\cdot)$ tends weak * to $U(\cdot)(\Phi(E))(\cdot)$, and the proof of the theorem is complete.

\section{References}

[1] Stefan Banach, Théorie des opérations Zinéaires (Monografje Matematyczne, 1. Z Subwencji Funduszu Kultury Narodowey, Warszawa, 1932).

[2] Michael Cambern, "The isometries of $L^{p}(X, K)$ ", Pacific J. Math. 55 $(1974), 9-17$.

[3] Michael Cambern, "On mappings of spaces of functions with values in a Banach space", Duke Math. J. 42 (1975), 91-98.

[4] Nicolae Dinculeanu, Vector measures (International Series of Monographs in Pure and Applied Mathematics, 95. Pergamon Press, Oxford, New York, Toronto; VEB Deutscher Verlag der Wissenschaften, Berlin, 1967).

[5] J.L. Doob, Stochastic processes (John Wiley \& Sons, New York; Chapman and Hall, London; 1953). 
[6] R.J. Fleming and J.E. Jamison, "Classes of operators on vector valued integration spaces", J. Austral. Math. Soc. Ser. A 24 (1977), 129-138.

[7] Kenneth Hoffman and Ray Kunze, Linear algebra, Second edition (Prentice-Hall, Englewood Cliffs, New Jersey, 1971).

[8] John Lamperti, "On the isometries of certain function-spaces", Pacific J. Math. 8 (1958), 459-466.

[9] A.R. Sourour, "The isometries of $L^{p}(\Omega, X)$ ", J. Funct. Anal. 30 $(1978), 276-285$.

[10] Angus E. Taylor, Introduction to functional analysis (John Wiley \& Sons, New York; Chapman and Hall, London, 1958).

\footnotetext{
Department of Mathematics,

University of California,

Santa Barbara,

California 93106,

USA.
} 